\title{
Pituitary tumor transforming gene binding factor (PBF) is required for androgen-induced prostate cancer proliferation and invasion
}

\author{
S. Q. HUANG ${ }^{1, *}$, B. WANG ${ }^{2, *}$, Q. J. LIAO ${ }^{3}$, C. X. SHEN ${ }^{1}$, W. B. LI' \\ ${ }^{1}$ Department of Urology and Nephrology, The Third Affiliated Hospital, Chongqing Medical University, Chongqing, China; ${ }^{2}$ Department of Urol- \\ ogy, The Fourth People's Hospital of Xi'an, Xian, China; ${ }^{3}$ Xinqiao Community Health Service Center of Shapingba District, Chongqing, China \\ ${ }^{*}$ Correspondence: $e$-mail: liweibing2018@163.com \\ "Contributed equally to this work.
}

Received July 30, 2018 / Accepted October 22, 2018

\begin{abstract}
Pituitary tumor transforming gene binding factor $(\mathrm{PBF})$ is a proto-oncogene that has a role in many cancers. However, its involvement in prostate cancer (PCa) remains unclear. Herein, we examined PBF expression in clinical specimens and investigated its regulation and function in human PCa cell lines. Immunohistochemical staining of patient tissues revealed higher $\mathrm{PBF}$ expression in PCa than in benign prostatic hyperplasia or adjacent normal prostate specimens. In LNCaP and $22 \mathrm{Rv} 1$ cells, PBF expression was upregulated by androgen treatment in a manner partially blocked by the androgen receptor (AR) antagonist bicalutamide. We identified a novel androgen response element in the PBF gene promoter and demonstrated its functional relevance using luciferase reporter assays. Androgen treatment of LNCaP cells induced binding between the endogenous $\mathrm{AR}$ and the androgen response element in $\mathrm{PBF}$, as measured by chromatin immunoprecipitation assays. Finally, RNA interference of PBF expression significantly reduced androgen-induced LNCaP cell growth and invasion. Thus, $\mathrm{PBF}$ is a novel AR target gene and has a role in androgen-induced proliferation and metastatic functions in PCa cells.
\end{abstract}

Key words: PBF, prostate cancer, cell proliferation, cell migration, androgen-responsive gene

Androgens play an important role in maintaining normal function of the prostate [1] and also play a key role in the development of prostate cancer (PCa) [2]. Androgens exert their molecular and cellular effects by binding to the androgen receptor (AR), which induces translocation of the AR to the nucleus, dimerization, formation of a complex with co-activators or co-repressors, and binding to specific DNA sequences known as androgen response elements (AREs). AREs are located in the promoter region of target genes and regulate expression of the androgen-responsive genes $[3,4]$. The growth, proliferation, and differentiation of PCa cells are affected by various androgen-responsive genes and the overall balance between the growth inhibitory and stimulatory effects of the genes determines the development and progression of PCa [5-7]. Therefore, identification and characterization of AR target genes is critical to advance our understanding of the role of androgens in PCa and to find novel therapeutic targets for this disease.

Pituitary tumor transforming gene binding factor (PBF) is a poorly characterized proto-oncogene that was originally identified through its ability to interact with PTTG1 and is also known as securin. PBF comprises six exons spanning $24 \mathrm{~kb}$ located on chromosome 21q22.3. The 180-amino acid peptide sequence of PBF has a predicted molecular mass of $22 \mathrm{kDa}$ and shares no significant homology with other human proteins, suggesting both unique functionality and significant evolutionary importance [8]. PBF is highly expressed in many tumors $[9,10]$. In breast cancer, PBF expression correlates with estrogen receptor positivity and its mRNA and protein levels are regulated by diethylstilbestrol and $17 \beta$-estradiol. In the breast cancer cell line MCF7, PBF upregulation significantly increases cell invasion in vitro, which is highly dependent on PBF secretion. In addition, repression of $\mathrm{PBF}$ expression prevents estrogen-induced MCF7 cell invasion [11]. PBF mRNA levels are higher in thyroid carcinomas than in normal thyroid tissues, and its expression can be used to both predict prognosis and identify high-risk patients for more aggressive treatment [12]. PBF is also overexpressed in colorectal cancer, particularly in invasive wild-type p53 and mutant p53 tumors, where it acts as a novel interacting partner of p53 and modulates its transactivation capabilities by altering p 53 stability and disrupting 
promoter binding. This implies that PBF could be a novel etiological marker in colorectal tumorigenesis [13]. Despite these observations, there have been no reports of a relationship between PBF and PCa.

In the present study, we compared the expression of $\mathrm{PBF}$ in human normal prostate, benign prostate hyperplasia $(\mathrm{BPH})$ and PCa specimens. Using the androgen-dependent human prostate cell lines LNCaP and 22Rv1, we also examined the effects of androgen treatment and AR activity on PBF expression. Finally, we determined how RNA interference-mediated knockdown (KD) of PBF affected PCa growth and invasion.

\section{Materials and methods}

Cell culture. Human LNCaP and 22Rv1 PCa cells were obtained from American Type Culture Collection (Manassas, VA, USA). Cells were maintained in RPMI 1640 medium supplemented with $10 \%$ fetal bovine serum (FBS) and $1 \%$ penicillin-streptomycin (both from Gibco-Life Technologies, USA) at $37^{\circ} \mathrm{C}$ in $5 \% \mathrm{CO}_{2}$ in air. For experiments with dihydrotestosterone (DHT) or bicalutamide, LNCaP and 22Rv1 cells were cultured in phenol red-free RPMI 1640 supplemented with 5\% steroid-depleted FBS for $48 \mathrm{~h}$ to ensure ligand-free conditions.

Knockdown of PBF. To obtain a stable PBF KD cell line, LNCaP cells were infected with recombinant lentivirus expressing PBF-specific shRNA sequence (Genema, China) together with luciferase and green fluorescent protein (GFP) under the control of CMV promoter. LNCaP control cells were infected with lentivirus expressing luciferase and GFP alone. Stable cell lines were selected as GFP+ cells by flow cytometry and $\mathrm{KD}$ of PBF protein expression was confirmed by western blotting of the resulting $\mathrm{LNCaP} / \mathrm{shPBF}$ and LNCaP/Control cell lines.

Clinical samples and immunohistochemical (IHC) staining. Matched specimens of PCa and adjacent normal and $\mathrm{BPH}$ tissue were collected from 12 patients at the Department of Urology \& Nephrology, The Third Affiliated Hospital, Chongqing Medical University, China. The study was approved by the Institutional Review Board of Chongqing Medical University, China and was performed in accordance with the provisions of the Declaration of Helsinki [14]. Written informed consent was obtained from all patients. IHC staining of PBF and calculation of an immunoreactivity score to quantify protein expression were both performed as previously described [15]. The primary anti-PBF antibody (sc-134414; Santa Cruz Biotechnology, USA) was used at a dilution of 1:200.

Western blot analysis. Cells were lysed in RIPA buffer and equal amounts of protein were separated by SDS-PAGE and then transferred to PVDF membranes (Merck Millipore, Germany). After blocking for $2 \mathrm{~h}$ at room temperature in 5\% milk in phosphate-buffered saline (PBS) $/ 0.1 \%$ Tween 20 , the membranes were incubated overnight at $4{ }^{\circ} \mathrm{C}$ with anti-PBF antibody (sc-134414; Santa Cruz Biotechnology) or anti- $\alpha-$ tubulin antibody (Beyotime, China). The membranes were then washed, incubated with the appropriate horseradish peroxidase-conjugated secondary antibodies and washed again. Finally, immunoreactive proteins were visualized with an Image Quant LAS-4000 BioImaging System (GE Healthcare, Sweden).

Quantitative RT-PCR. Total RNA was extracted using TRIzol reagent (Takara, China) and reverse transcribed to cDNA using a PrimeScript ${ }^{\mathrm{TM}} \mathrm{RT}$ Reagent Kit (Takara) according to the manufacturer's protocols. The primers used were: PBF forward 5'-CTCTTCTCAGTTTGTGAAACGCTAA-3', PBF reverse 5'-CTGCCCTGGGAGAATGACA-3'; glyceraldehyde 3-phosphate dehydrogenase (GAPDH) forward 5'-CGCTGAGTACGTCGTGGAGTC-3', GAPDH reverse 5'-GCTGATGATCTTGAGGCTGTTGTC-3'. Quantitative RT-PCR was performed with SYBR Green Realtime PCR Master Mix (Toyobo, Osaka, Japan) using a StepOnePlus Real-Time PCR System (Life Technologies, USA). The thermocycling conditions were: $95^{\circ} \mathrm{C}$ for $1 \mathrm{~min}, 95^{\circ} \mathrm{C}$ for $15 \mathrm{~s}, 60^{\circ} \mathrm{C}$ for $15 \mathrm{~s}$, and $72^{\circ} \mathrm{C}$ for $45 \mathrm{~s}$ (40 cycles). GAPDH expression levels were used to normalize RNA input differences.

Luciferase assays. For the luciferase reporter assays, three PBF promoter fragments $(-2000 /+1,-1500 /+1,-1000 /+1)$ were amplified using the primers 5'-GAAGATCTAGAGCCAGCGTTTTCCAATTGAGC-3', 5'-GAAGATCTGAAATAACAAACGAAACTCTTGCCTT-3', and 5'-GAAGATCTACGGTTTCCCGTCTTCTGGTCTA-3', respectively as forward primers and 5'-GGTCGGCCGGTCGCTCTAT-3' as reverse primer. The fragments were cloned into the PmirGLO-P luciferase reporter vector (Promega, USA) to give plasmids Pmir/-2000, Pmir/-1500 and Pmir/-1000. The mutated promoter plasmid (Mut-Pmir/-1000) was generated using 5'-ACGTAAAAAACTAGGATCGTGTAAT-3' (forward) and 5'-TTTTTACGTGCCACAAAGTTTGCAAG-3' (reverse) primers. Cells were plated in 24-well plates, grown to $70-80 \%$ confluence and then transfected with plasmids $(0.5 \mu \mathrm{g})$ using Lipofectamine 2000 transfection reagent (Invitrogen) according to the manufacturer's instructions. After $24 \mathrm{~h}$ incubation, whole-cell lysates were prepared for detection of luciferase activity using the Dual Luciferase Reporter System (Promega).

Chromatin immunoprecipitation (ChIP) assays. LNCaP and 22Rv1 cells were treated with $1 \mathrm{nM}$ DHT or ethanol vehicle for $48 \mathrm{~h}$. The cells $\left(10^{7} /\right.$ sample) were collected, crosslinked and lysed using a ChIP-IT Express Kit (Active Motif, USA). Chromatin was sonicated to $200-800 \mathrm{bp}$ fragments with eight rounds of 10 -s pulses using $25 \%$ power. Normalized inputs of sheared chromatin DNA were incubated with $4 \mathrm{mg}$ of IgG (negative control) or anti-AR antibody (ab74272, Abcam, UK) overnight at $4{ }^{\circ} \mathrm{C}$. The immunocomplexes were collected and subjected to Q-PCR using the following primers: PBF forward 5'-TTAGCAGGACCGCGGCGTCCTT-3' and reverse 5'-CGGTGCCGGGGACAGAAACCT-3', and prostate-specific antigen (PSA) forward 5'-CTGCCTTT- 
GTCCCCTAGATG-3' and reverse 5'-TATGCTGGAGGCTGGACAAC-3'

Clonogenic assays. $\mathrm{LNCaP} / \mathrm{shPBF}$ and $\mathrm{LNCaP} /$ Control cells were plated in 6-well plates at $10^{3}$ cells/dish and cultured for 14 days. The medium was changed after 7 days. On day 14, colonies were stained with $0.5 \%$ crystal violet in $4 \%$ formaldehyde for $30 \mathrm{~min}$ and the number of colonies was counted under a microscope.

Transwell invasion assay. Aliquots of $1 \times 10^{5}$ cells in phenol red-free medium were seeded into the upper chambers of a Transwell insert (Costar, USA) pre-coated with Matrigel (BD Biosciences, USA). Phenol red-free RPMI 1640 medium supplemented with $5 \%$ steroid-depleted FBS with or without $1 \mathrm{nM}$ DHT was placed in the lower chambers. The cells were cultured for $48 \mathrm{~h}$, and invaded cells on the lower surface of the insert were then stained with $0.1 \%$ crystal violet and counted under a microscope.

Cell proliferation assay. Proliferation was assessed using the MTT-based CellTiter 96 Non-Radioactive Cell Proliferation Assay Kit (Promega) according to the manufacturer's protocol. $\mathrm{LNCaP} / \mathrm{shPBF}$ and $\mathrm{LNCaP} /$ Control cells were re-suspended in phenol red-free RPMI 1640 containing 5\% steroid-depleted FBS with or without $1 \mathrm{nM}$ DHT, plated in 96-well plates at $2 \times 10^{3}$ cells/well and incubated at $37^{\circ} \mathrm{C}$. Cell viability was measured each day for 7 days. In this assay, absorbance at $490 \mathrm{~nm}$ is proportional to the number of living cells.

Statistical analysis. All analyses were conducted using SPSS 13.0 software. Data are expressed as the mean \pm standard deviation (SD). Expression of PBF in clinical specimens was evaluated using the Mann-Whitney test. For all other experiments, differences were analyzed by ANOVA or Student's t-test. A p-value of $<0.05$ was considered statistically significant.

\section{Results}

Identification of $\mathrm{PBF}$ expression in prostate cancer tissues. PBF expression in 12 matched samples of $\mathrm{PCa}, \mathrm{BPH}$ and adjacent normal tissue was examined by IHC. We found that PBF expression was higher in PCa than in the adjacent normal and $\mathrm{BPH}$ tissues (Figure 1).

Androgens regulate PBF expression in PCa cells. To determine whether PBF expression is directly regulated by androgens, we treated androgen-sensitive human PCa cell lines LNCaP and 22Rv1 with DHT in presence or absence of the AR antagonist bicalutamide. Western blotting and Q-RT-PCR analysis revealed that DHT significantly increased $\mathrm{PBF}$ protein and mRNA expression, respectively, and this was significantly attenuated by bicalutamide (Figures 2A, $2 \mathrm{~B}$, and $2 \mathrm{E}$ ). Similar results were obtained using $22 \mathrm{Rv} 1$ cells (Figure 2C-E). These results indicate that PBF expression is upregulated by androgen and suggest that it occurs in an AR-dependent manner.

Androgen increased PBF expression by activating the AR. To verify the involvement of the AR in DHT-induced PBF upregulation, we performed luciferase reporter assays. Luciferase plasmids containing fragments of the PBF promoter $(-2000 /+1,-1500 /+1$, and $-1000 /+1)$ were transfected into LNCaP and 22Rv1 cells, which were then treated with vehicle or DHT for $24 \mathrm{~h}$. High levels of luciferase
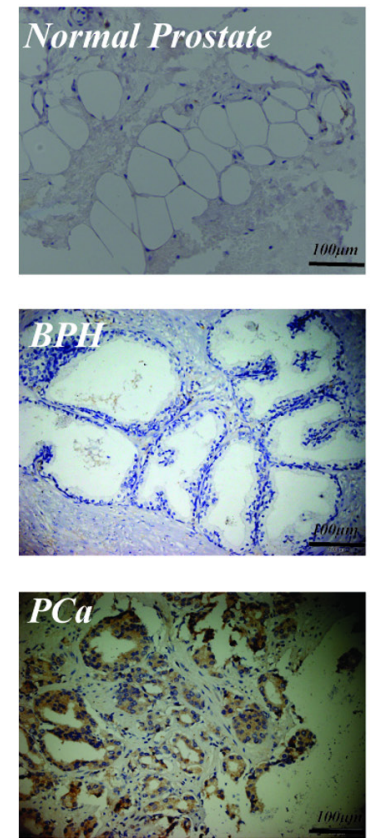

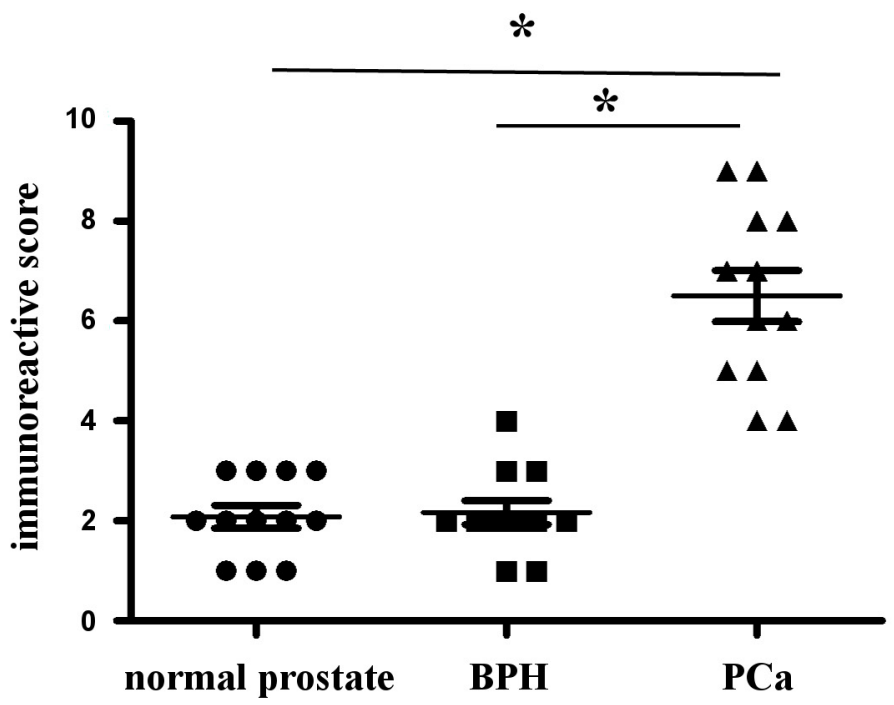

Figure 1. PBF expression in prostate cancer specimens. Immunohistochemical analysis of 12 matched clinical samples of PCa and adjacent normal and BPH tissues. Data are mean $\pm S D .{ }^{\star} p<0.05,{ }^{\star *} p<0.01$. 
activity were detected in DHT-stimulated cells transfected with the $-2000 /+1$ and $-1500 /+1$ plasmids, but not with the $-1000 /+1$ plasmid (Figure 3A, 3B). These data indicate that at least one ARE is localized between -1500 and -1000 of the PBF promoter.

We then analyzed the regulatory regions of the PBF gene using the web-based tool ConSite, which enables the discovery of cis-regulatory elements in genomic sequences. ConSite analysis identified one putative AR-binding site in the -1500 to -1000 sequence of the promoter (Figure 3C). To confirm the presence of an ARE, we repeated the luciferase reporter assay using a construct in which we mutated the putative ARE in the -1500 to -1000 region. As expected,
DHT-induced luciferase activity was significantly suppressed in cells expressing the mutated promoter compared to the wild-type construct (Figures 3D, 3E). To verify AR-ARE binding in PCa cells, we performed ChIP assays in which cross-linked chromatin from LNCaP and 22Rv1 cells was immunoprecipitated with an anti-AR antibody, after which the immunocomplexed material was analyzed for the $\mathrm{PBF}$ promoter -1500 to -1000 sequence by PCR. As shown in Figure $3(\mathrm{~F}-\mathrm{H})$, the putative ARE in the PBF promoter from DHT-stimulated LNCaP and 22Rv1 cells prostate cells was immunoprecipitated by an anti-AR antibody, but not control IgG. These data indicate that DHT treatment induces binding of the endogenous AR to the PBF promoter in PCa cells.
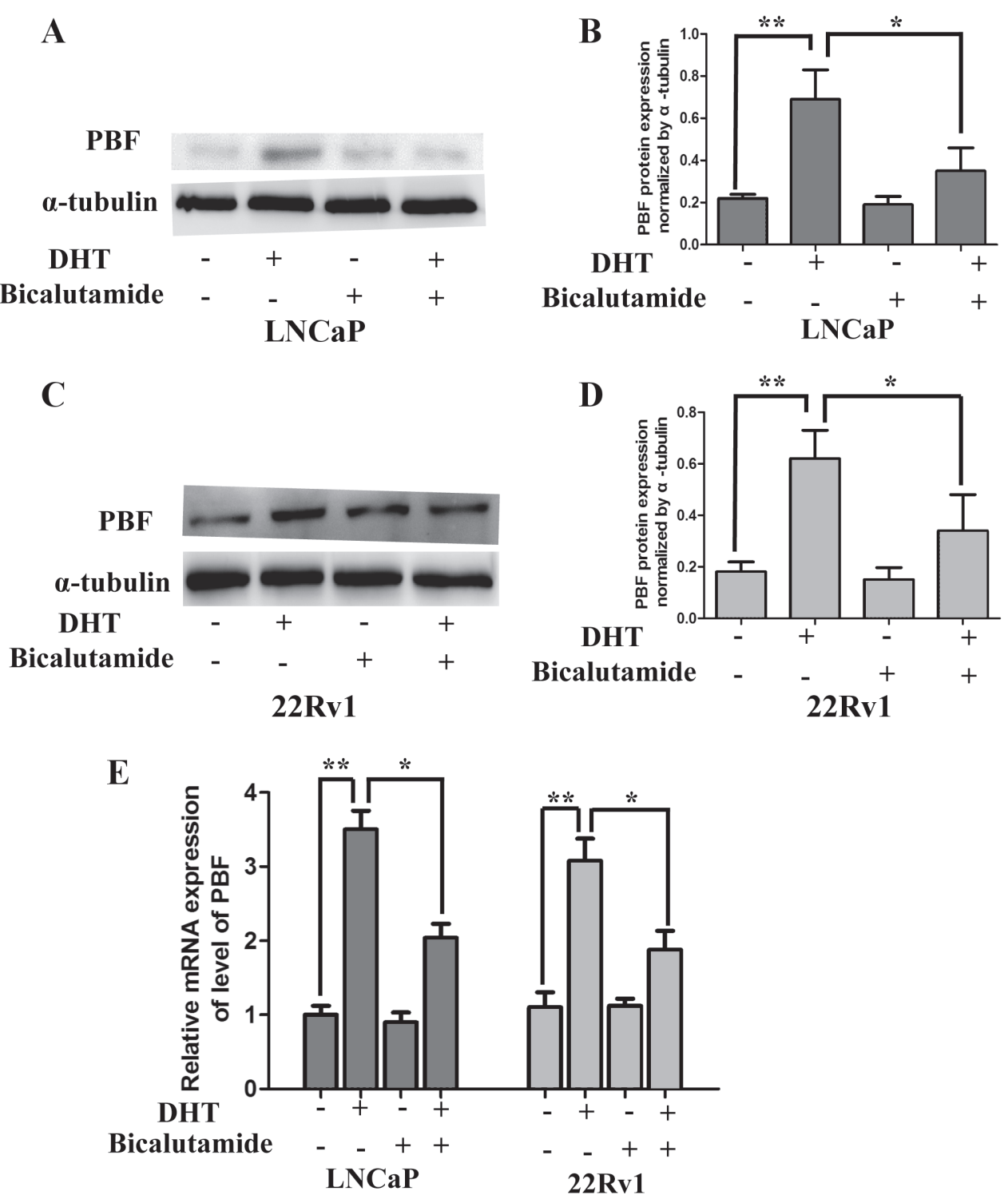

Figure 2. PBF regulation by androgen in prostate cancer $\mathrm{LNCaP}$ and 22Rv1 cells is dependent on the androgen receptor. Western blot analysis of PBF expression in LNCaP cells (A, B) and 22Rv1 cells (C, D). E) Quantitative RT-PCR analysis of PBF mRNA expression in LNCaP cells and 22Rv1 cells. Data are mean $\pm \mathrm{SD} .{ }^{*} \mathrm{p}<0.05,{ }^{* *} \mathrm{p}<0.01$. 
PBF is required for androgen-induced PCa cell growth and invasion. To explore the effects of PBF on androgeninduced $\mathrm{PCa}$ cell growth, we examined the viability of $\mathrm{LNCaP} / \mathrm{shPBF}$ and LNCaP/Control cells cultured with or without DHT each day for 7 days using the MTT assay. As shown in Figure 4B, PBF KD did not affect the growth of unstimulated cells; however, it dramatically reduced it in cells exposed to DHT, and a significant difference was detected after 5 days of growth. Similar results were obtained when the proliferative capacity of PCa cells was examined using a
A

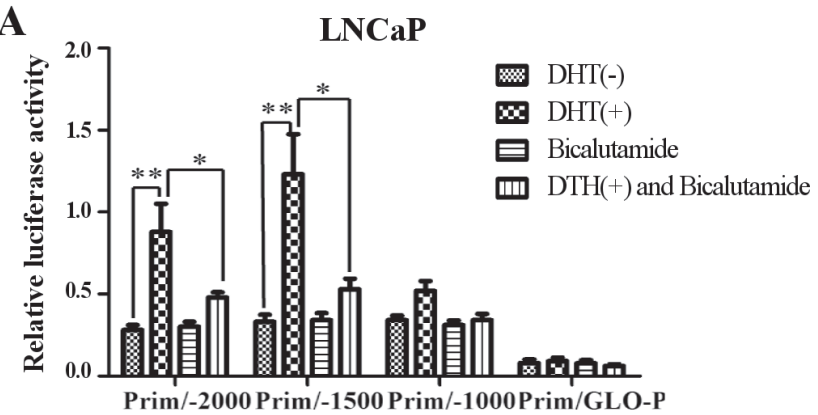

C

पpotential AR bind site

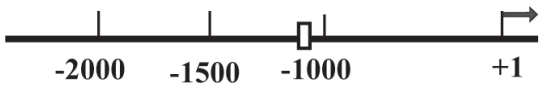

$\mathbf{E}$

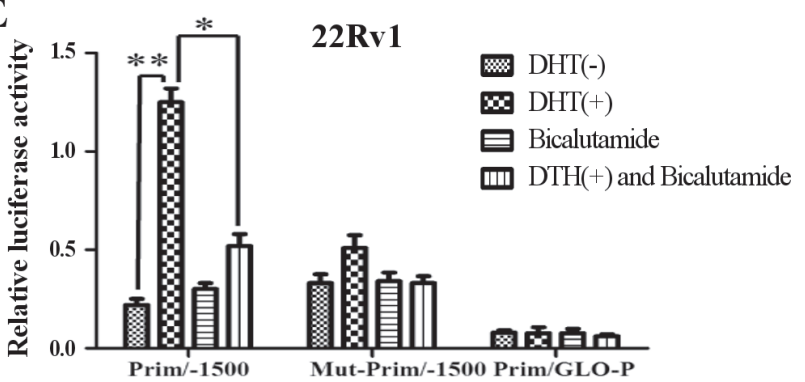

G

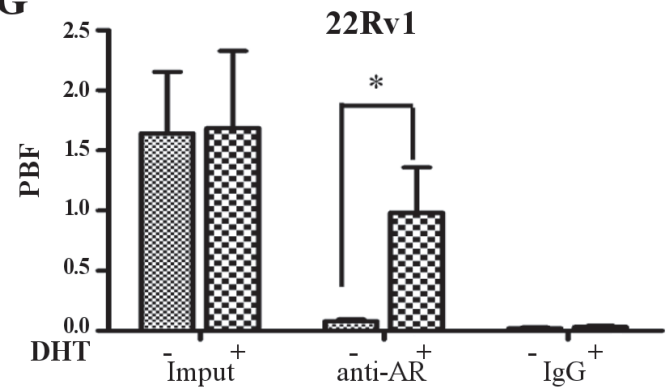

B

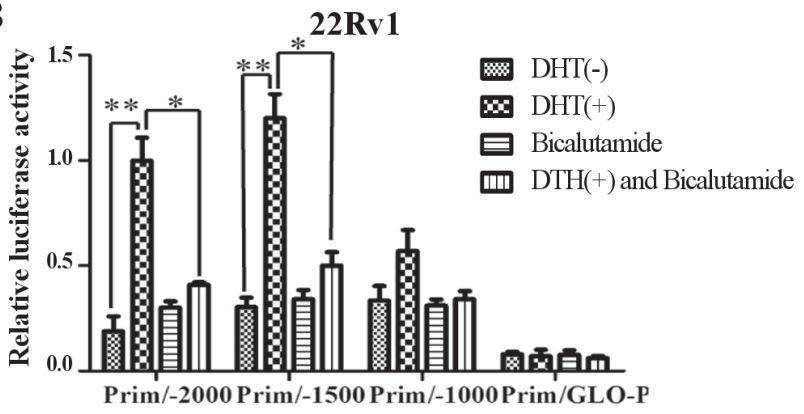

D

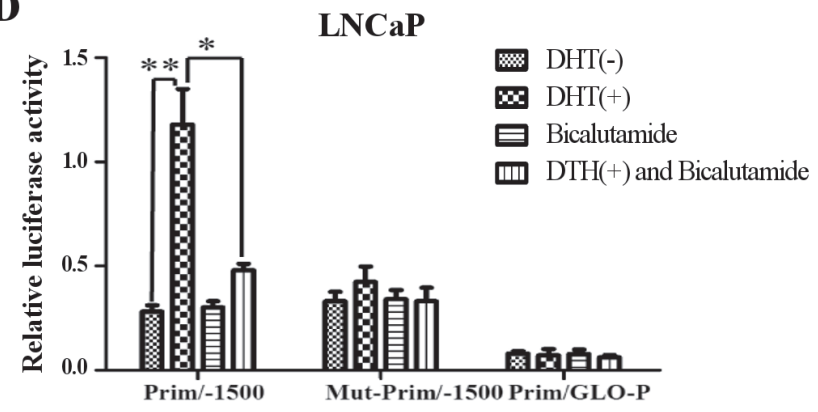

$\mathbf{F}$
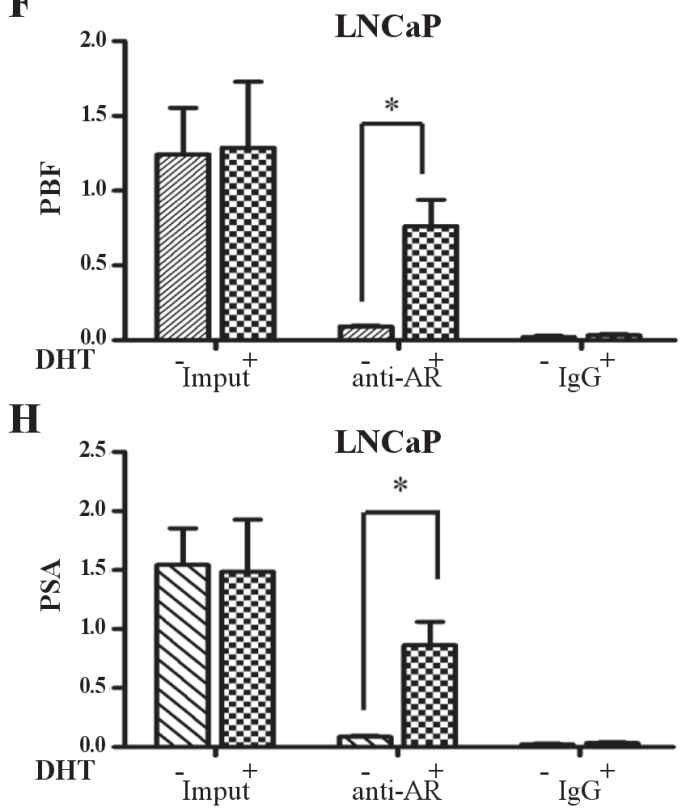

Figure 3. Identification of an androgen response element in the PBF gene promoter. A, B) Luciferase reporter assays driven by PBF promoter constructs of various lengths in LNCaP and 22Rv1 cells. C) Schematic representation of potential AR-binding sites in the PBF promoter. D, E) Quantitation of luciferase activity driven by wild-type and ARE-mutant PBF promoters in LNCaP cells and 22Rv1 cells. F, G) ChIP analysis of a putative AR-binding site in the PBF promoter. Extracts from LNCaP cells and 22Rv1 cells were immunoprecipitated with control IgG or anti-AR antibody, and PBF promoter sequences in the immunoprecipitates were detected by PCR. H) ChIP analysis of the AR-binding site in the PSA promoter (positive control). Data are mean \pm SD. ${ }^{*} \mathrm{p}<0.05,{ }^{*} \mathrm{p}<0.01$. 
A

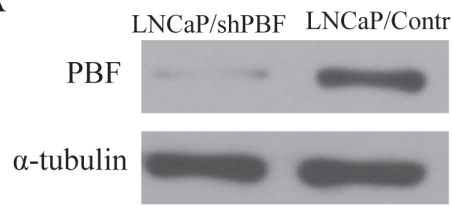

B

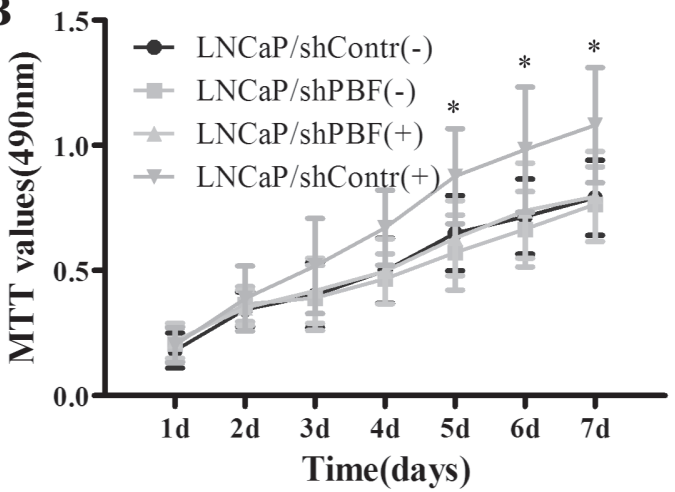

C

D
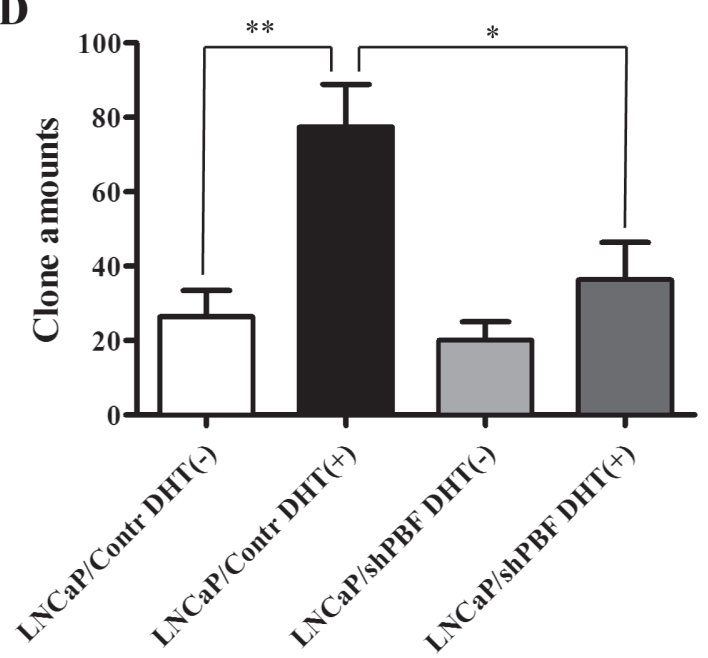

E

\section{F}
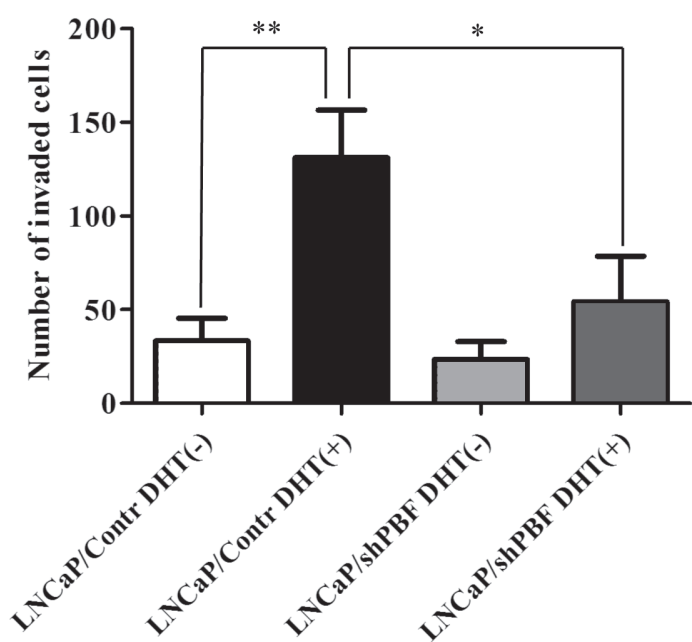

Figure 4. Effects of PBF knock-down on LNCaP cell growth and invasiveness. A) Western blot analysis of PBF in LNCaP cells expressing empty vector (control) or PBF-specific shRNA. B) cell viability measured by MTT assay in the presence of DHT. C) Representative results of a clonogenic assay of $\mathrm{LNCaP} /$ Control and LNCaP/shPBF cells and quantification of colonies (D) per low-power field. E) Transwell invasion assays of LNCaP/Control and $\mathrm{LNCaP} / \mathrm{sh} \mathrm{PBF}$ cells and quantification of invaded cells $(\mathrm{F})$. Data are the mean $\pm \mathrm{SD} .{ }^{\star} \mathbf{p}<0.05,{ }^{* *} \mathrm{p}<0.01$. 
colony-forming assay (Figures 4C, 4D). To analyze PCa cell invasion, we performed a Transwell assay in which FBS was added to the lower wells as a chemoattractant. As shown in Figure 4 (E and $\mathrm{F})$, there was no significant difference in invasion of $\mathrm{LNCaP} / \mathrm{shPBF}$ and $\mathrm{LNCaP} /$ Control cells in the absence of DHT. Notably, although DHT significantly increased the number of migrating LNCaP/Control cells, KD of PBF significantly reduced this DHT-stimulated response. Taken together, these data suggest that PBF expression contributes to the stimulatory effects of androgen on PCa cell growth and invasion.

\section{Discussion}

Although PBF has been studied in other cancers, little is known about its expression and function in PCa. In the present study, we found that PBF is highly expressed in clinical PCa specimens, suggesting its possible involvement in disease pathogenesis. Using androgen-dependent $\mathrm{PCa}$ cell lines, we verified the upregulation of PBF by androgen, demonstrated the existence of an ARE in the promoter region of the $\mathrm{PBF}$ gene, and verified that endogenous $\mathrm{AR}$ binds to the PBF ARE in PCa cells, thereby establishing the molecular mechanism underlying androgen-dependent transcriptional upregulation of PBF in PCa. Furthermore, we demonstrated the functional relevance of these observations by showing that PBF KD attenuated androgeninduced proliferation and invasion by PCa cells. Collectively, these results suggest not only that $\mathrm{PBF}$ is a direct target gene of AR but also that it plays a key role in androgendependent $\mathrm{PCa}$.

Studies on the genesis and progression of PCa have mainly focused on the activation and expression of oncogenes [16, 17]. $\mathrm{PBF}$ is a multifunctional glycoprotein that is overexpressed in a wide range of tumors and is significantly associated with poorer oncological outcomes, such as early tumor recurrence, distant metastasis, extramural vascular invasion and decreased disease-specific survival [18]. Our study found that PBF is expressed at higher levels in PCa tissues compared with adjacent normal and $\mathrm{BPH}$ tissues, implying that it may play a crucial role in human PCa.

Androgens have a well-known role in maintaining prostate morphology and function and additionally in the development of PCa [19]. We explored the effects of DHT on PBF and found that it upregulates PBF expression in LNCaP and 22Rv1 cells, and this effect was attenuated by the AR antagonist bicalutamide. Like many steroid hormone receptors, AR is a cytoplasmic protein which translocates to the nucleus upon ligand binding and then binds to AREs to modulate gene transcription [20, 21]. A variety of ARE consensus sequences have been identified in multiple AR-regulated genes, typically containing a core sequence of GGTACAnnnTGTTCT and requiring at least three guanines [22]. Using the web-based tool ConSite, we identified a putative cis-regulatory element in the PBF promoter at -1073 to -1094 that had sequence homology to typical AREs. Indeed, we demonstrated that the -1500 to -1000 sequence of PBF, which contained the putative ARE, responded strongly to DHT in a luciferase reporter assay. We confirmed by ChIP assays that endogenous AR protein in PCa cells binds to the PBF promoter in the presence of androgen. These data clearly demonstrate that $\mathrm{PBF}$ is an androgen-regulated gene in LNCaP and 22Rv1 cells. Because bicalutamide did not completely block androgeninduced PBF expression, it is probable that other unknown factors also take part in the androgen-induced regulation of PBF expression in PCa cells. A number of AR co-regulators can play auxiliary activating, inhibiting, or dual roles in AR-mediated transcription [23], and some may affect androgen-regulated expression of PBF. This will be investigated in a future study.

As an established proto-oncogene, PBF plays important roles in the growth of several tumors. For example, PBF promotes proliferation and invasion of colorectal cancer cells [13], while its expression in normal thyroid cells can lead to hyperplasia and adenoma formation. In thyroid cancer, PBF promotes growth and invasive ability of tumor cells, and its expression appears to be associated with tumor stage and metastasis [24-26]. Estrogen treatment of post-menopausal women with breast cancer may upregulate the expression and secretion of PBF, leading to increased cell invasion [11]. In our study, we performed shRNA-mediated KD of PBF to investigate its contribution to $\mathrm{PCa}$ growth and found that it is necessary for androgen-induced growth and invasion of LNCaP cells. Although the mechanism by which this may occur is not clear, one possibility is that PBF promotes the nuclear localization of PTTG1; indeed, PBF was identified through its interaction with this protein [27]. In our previous study we found that PTTG1 is highly expressed in PCa and can promote cell proliferation and invasion $[28,29]$. Thus, PBF may facilitate the function of PTTG1 in promoting PCa proliferation and metastasis. However, some studies suggest that PBF can act independently of PTTG1. In colorectal and thyroid cancers, PBF promotes tumor development and metastasis by regulating p53 activity $[13,30]$. Further work will be required to understand the precise mechanism by which PBF contributes to the androgen effects on cancer cell growth and invasion.

In conclusion, our results shed light on the mechanisms of androgen-induced PBF expression and its functional consequences for PCa progression. In addition, we have demonstrated the pattern of PBF expression in normal prostate, $\mathrm{BPH}$ and PCa tissues in humans.

Acknowledgments: This work was supported by an incubation project grant (KY08033) of The Third Affiliated Hospital, Chongqing Medical University (Gener Hospital). We thank Anne M. O'Rourke, PhD, from Liwen Bianji, Edanz Group China (www. liwenbianji.cn/ac) for editing the English text of a draft of this manuscript. 


\section{References}

[1] LA VIGNERA S, CONDORELLI RA, RUSSO GI, MORGIA G, CALOGERO AE. Endocrine control of benign prostatic hyperplasia. Andrology 2016; 4: 404-411. https://doi. org/10.1111/andr.12186

[2] PARSONS TK, PRATT RN, TANG L, WU Y. An active and selective molecular mechanism mediating the uptake of sex steroids by prostate cancer cells. Mol Cell Endocrinol 2018; 477: 121-131. https://doi.org/10.1016/j.mce.2018.06.009

[3] FAJARDO AM, MACKENZIE DA, OLGUIN SL, SCARIANO JK, RABINOWITZ I et al. Antioxidants Abrogate AlphaTocopherylquinone-Mediated Down-Regulation of the Androgen Receptor in Androgen-Responsive Prostate Cancer Cells. PLoS One 2016; 11: e0151525. https://doi.org/10.1371/ journal.pone.0151525

[4] EDER IE, CULIG Z, PUTZ T, NESSLER-MENARDI C, BARTSCH $\mathrm{G}$ et al. Molecular biology of the androgen receptor: from molecular understanding to the clinic. Eur Urol 2001; 40: 241-251. https://doi.org/10.1159/000049782

[5] DEHM SM, TINDALL DJ. Regulation of androgen receptor signaling in prostate cancer. Expert Rev Anticancer Ther 2005; 5: 63-74. https://doi.org/10.1586/14737140.5.1.63

[6] VAZ CV, MAIA CJ, MARQUES R, GOMES IM, CORREIA $S$ et al. Regucalcin is an androgen-target gene in the rat prostate modulating cell-cycle and apoptotic pathways. Prostate 2014; 74: 1189-1198. https://doi.org/10.1002/pros.22835

[7] JIANG F, WANG Z. Identification of androgen-responsive genes in the rat ventral prostate by complementary deoxyribonucleic acid subtraction and microarray. Endocrinology 2003; 144: 1257-1265. https://doi.org/10.1210/en.2002220718

[8] SMITH VE, FRANKLYN JA, MCCABE CJ. Pituitary tumortransforming gene and its binding factor in endocrine cancer. Expert Rev Mol Med 2010; 12: e38. https://doi.org/10.1017/ S1462399410001699

[9] WANG XP, DENG XL, LI LY. MicroRNA-584 functions as a tumor suppressor and targets PTTG1IP in glioma. Int J Clin Exp Pathol 2014; 7: 8573-8582.

[10] WATKINS RJ, IMRUETAICHAROENCHOKE W, READ ML, SHARMA N, POOLE VL et al. Pro-invasive Effect of Proto-oncogene PBF Is Modulated by an Interaction with Cortactin. J Clin Endocrinol Metab 2016; 101: 4551-4563. https://doi.org/10.1210/jc.2016-1932

[11] WATKINS RJ, READ ML, SMITH VE, SHARMA N, REYNOLDS GM et al. Pituitary tumor transforming gene binding factor: a new gene in breast cancer. Cancer Res 2010; 70: 3739-3749. https://doi.org/10.1158/0008-5472.CAN-093531

[12] STRATFORD AL, BOELAERT K, TANNAHILL LA, KIM DS, WARFIELD A et al. Pituitary tumor transforming gene binding factor: a novel transforming gene in thyroid tumorigenesis. J Clin Endocrinol Metab 2005; 90: 4341-4349. https://doi.org/10.1210/jc.2005-0523

[13] READ ML, SEED RI, MODASIA B, KWAN PP, SHARMA $\mathrm{N}$ et al. The proto-oncogene PBF binds p53 and is associated with prognostic features in colorectal cancer. Mol Carcinog 2016; 55: 15-26. https://doi.org/10.1002/mc.22254
[14] WORLD MEDICAL ASSOCIATION. World Medical Association Declaration of Helsinki: ethical principles for medical research involving human subjects. JAMA 2013; 310: 2191-2194. https://doi.org/10.1001/jama.2013.281053

[15] DIND X, YANG DR, XIA L, CHEN B, YU S et al. Targeting TR4 nuclear receptor suppresses prostate cancer invasion via reduction of infiltrating macrophages with alteration of the TIMP-1/MMP2/MMP9 signals. Mol Cancer 2015; 14: 16. https://doi.org/10.1186/s12943-014-0281-1

[16] EAGLELR, YINX, BROTHMAN AR, WILLIAMS BJ, ATKIN $\mathrm{NB}$ et al. Mutation of the MXI1 gene in prostate cancer. Nat Genet 1995; 9: 249-255. https://doi.org/10.1038/ng0395-249

[17] PORKKA KP, VISAKORPI T. Molecular mechanisms of prostate cancer. Eur Urol 2004; 45: 683-691. https://doi. org/10.1016/j.eururo.2004.01.012

[18] IMRUETAICHAROENCHOKE W, FLETCHER A, LU W, WATKINS RJ, MODASIA B et al. Functional consequences of the first reported mutations of the proto-oncogene PTTG1IP/PBF. Endocr Relat Cancer 2017; 24: 459-474. https:// doi.org/10.1530/ERC-16-0340

[19] YADAV N, HEEMERS HV. Androgen action in the prostate gland. Minerva Urol Nefrol 2012; 64: 35-49.

[20] TILKID,SCHAEFFEREM,EVANSCP.Understanding Mechanisms of Resistance in Metastatic Castration-resistant ProstateCancer:TheRoleofthe Androgen Receptor.EurUrolFocus 2016; 2: 499-505. https://doi.org/10.1016/j.euf.2016.11.013

[21] ShAFI AA, YEN AE, WEIGEL NL. Androgen receptors in hormone-dependent and castration-resistant prostate cancer. Pharmacol Ther 2013; 140: 223-238. https://doi. org/10.1016/j.pharmthera.2013.07.003

[22] ZhANG Z, JIN B, JIN Y, HUANG S, NIU X et al. PTTG1, A novel androgen responsive gene is required for androgen-induced prostate cancer cell growth and invasion. Exp Cell Res 2017; 350: 1-8. https://doi.org/10.1016/j.yexcr.2016.10.013

[23] ATTAR RM, TAKIMOTO CH, GOTTARDIS MM. Castration-resistant prostate cancer: locking up the molecular escape routes. Clin Cancer Res 2009; 15: 3251-3255. https:// doi.org/10.1158/1078-0432.CCR-08-1171

[24] READ ML, FONG JC, MODASIA B, FLETCHER A, IMRUETAICHAROENCHOKE $\mathrm{W}$ et al. Elevated PTTG and PBF predicts poor patient outcome and modulates DNA damage response genes in thyroid cancer. Oncogene 2017; 36: 5296 5308. https://doi.org/10.1038/onc.2017.154

[25] HSUEH C, LIN JD, CHANG YS, HSUEH S, CHAO TC et al. Prognostic significance of pituitary tumour-transforming gene-binding factor $(\mathrm{PBF})$ expression in papillary thyroid carcinoma. Clin Endocrinol (Oxf) 2013; 78: 303-309. https://doi.org/10.1111/cen.12007

[26] READ ML, LEWY GD, FONG JC, SHARMA N, SEED RI et al. Proto-oncogene PBF/PTTG1IP regulates thyroid cell growth and represses radioiodide treatment. Cancer Res 2011; 71: 6153-6164. https://doi.org/10.1158/0008-5472. CAN-11-0720

[27] XIANG C, GAO H, MENG L, QIN Z, MA R et al. Functional variable number of tandem repeats variation in the promoter of proto-oncogene PTTG1IP is associated with risk of estrogen receptor-positive breast cancer. Cancer Sci 2012; 103: 1121-1128. https://doi.org/10.1111/j.13497006.2012.02266.x 
[28] HUANG S, LIAO Q, LI L, XIN D. PTTG1 inhibits SMAD3 in prostate cancer cells to promote their proliferation. Tumour Biol 2014; 35: 6265-6270. https://doi.org/10.1007/s13277014-1818-Z

[29] HUANG SQ, LIAO QJ, WANG XW, XIN DQ, CHEN SX et al. RNAi-mediated knockdown of pituitary tumor- transforming gene-1 (PTTG1) suppresses the proliferation and invasive potential of PC3 human prostate cancer cells. Braz J Med Biol Res 2012; 45: 995-1001.
[30] READ ML, SEED RI, FONG JC, MODASIA B, RYAN GA et al. The PTTG1-binding factor (PBF/PTTG1IP) regulates p53 activity in thyroid cells. Endocrinology 2014; 155: 12221234. https://doi.org/10.1210/en.2013-1646 